\title{
Effectiveness of seven-valent pneumococcal conjugate vaccine against invasive pneumococcal disease: a matched case-control study
}

\author{
Cynthia G Whitney, Tamar Pilishvili, Monica M Farley, William Schaffner, Allen S Craig, Ruth Lynfield, Ann-Christine Nyquist, \\ Kenneth A Gershman, Marietta Vazquez, Nancy M Bennett, Arthur Reingold, Ann Thomas, Mary P Glode, Elizabeth R Zell, James H Jorgensen, \\ Bernard Beall, Anne Schuchat
}

\begin{abstract}
Summary
Background When seven-valent pneumococcal conjugate vaccine was introduced in the USA, many children were vaccinated on schedules that differed from those tested in clinical trials. Our aim was to assess the effectiveness of the vaccine against various pneumococcal serotypes, and to measure the effectiveness of the recommended dose schedule and of catch-up and incomplete schedules.
\end{abstract}

Methods Invasive disease, defined as isolation of pneumococcus from a sterile site, was identified in children aged 3-59 months through the US Centers for Disease Control and Prevention's Active Bacterial Core surveillance. We tested isolates for serotype and antimicrobial susceptibility. Three controls, matched for age and zip code were selected for each case. We calculated the matched odds ratio for vaccination using conditional logistic regression, controlling for underlying conditions. Vaccine effectiveness was calculated as one minus the adjusted matched odds ratio times $100 \%$.

Findings We enrolled 782 cases and 2512 controls. Effectiveness of one or more doses against vaccine serotypes was $96 \%(95 \%$ CI $93-98)$ in healthy children and $81 \%(57-92)$ in those with coexisting disorders. It was $76 \%(63-85)$ against infections that were not susceptible to penicillin. Vaccination prevented disease caused by all seven vaccine serotypes, and by vaccine-related serotype $6 \mathrm{~A}$. Several schedules were more protective than no vaccination; three infant doses with a booster were more protective against vaccine-type disease than were three infant doses alone $(\mathrm{p}=\mathbf{0} \cdot \mathbf{0 3 2 3})$.

Interpretation The seven-valent pneumococcal conjugate vaccine prevents invasive disease in both healthy and chronically ill children. The vaccine is effective when used with various non-standard schedules.

\section{Introduction}

Seven-valent pneumococcal conjugate vaccine was designed to prevent pneumococcal disease in young children. In randomised, blinded, controlled clinical trials of this vaccine and a closely related nine-valent version, the vaccines were highly effective against invasive infections, ${ }^{1-4}$ moderately so against pneumonia, ${ }^{3-5}$ and somewhat so against otitis media. ${ }^{6}$ On the basis of such evidence, the seven-valent vaccine was licensed to prevent pneumococcal disease in infants in the USA and was recommended for use in all children younger than 2 years and in children aged $2-4$ years in a high-risk category. ${ }^{7 .}$

After licensure, health-care providers began using the vaccine, with various schedules. Providers gave doses to infants at ages 2 months, 4 months, 6 months, and 12-15 months, according to the four-dose schedule developed in clinical trials of the seven-valent vaccine, and used several recommended but largely untested catch-up schedules ${ }^{7}$ in older infants and toddlers. Between August, 2001, and September, 2004, the seven-valent vaccine was often in short supply, and health-care providers were frequently unable to give the recommended number of doses. ${ }^{9}$ Nonetheless, surveil- lance data indicated that vaccine introduction substantially diminished the burden of invasive disease..$^{10-12}$

To assess the effectiveness of the seven-valent pneumococcal conjugate vaccine we did a matched casecontrol study. Our primary objective was to measure effectiveness of pneumococcal conjugate vaccine against invasive disease caused by various pneumococcal strains, including the seven vaccine serotypes in children 3-59 months old, and to assess effectiveness of various schedules.

\section{Methods}

\section{Study population}

Cases of invasive pneumococcal disease were identified through the Active Bacterial Core Surveillance (ABCS). This population and laboratory based surveillance system is operated by the US Centers for Disease Control and Prevention (CDC) as part of its Emerging Infections Program Network. ${ }^{13}$ The surveillance area was defined to include cases from San Francisco, California; Denver, Colorado; Connecticut state; Atlanta, Georgia; Minnesota (Minneapolis and Saint Paul in 2001 and the entire state from the beginning of 2002); Rochester and Albany, New York; Portland, Oregon; and 11 counties in Tennessee.
Lancet 2006; 368: 1495-502 See Comment page 1469

Division of Bacterial and Mycotic Diseases, National Center for Infectious Diseases, Centers for Disease Control and Prevention, Atlanta, GA, USA (CG Whitney MD,

TPilishvili MPH, E R Zell MStat, B Beall PhD, A Schuchat MD); Department of Medicine, Emory University, Veterans Affairs Medical Center, Atlanta, GA, USA (Prof M M Farley MD); Vanderbilt University School of Medicine, Nashville, TN, USA (Prof W Schaffner MD); Tennessee Department of Health, Nashville, TN, USA (A S Craig MD); Minnesota Department of Health, Minneapolis, MN, USA (R Lynfield MD); The Children's Hospital, Denver, CO, USA (A-C Nyquist MD, Prof M P Glode MD); Colorado Department of Public Health and Environment, Denver, $\mathrm{CO}$, USA (K A Gershman MD); Department of Pediatrics, Yale University, New Haven, CT, USA (M Vazquez MD); Department of Medicine, University of Rochester, Rochester, NY, USA (Prof N M Bennett MD); School of Public Health, University of California, Berkeley, CA, USA (Prof A Reingold MD); Oregon State Public Health Division, Portland, OR, USA (A Thomas MD); and University of Texas Health Sciences Center, San Antonio, TX, USA (ProfJ $\mathrm{H}$ Jorgensen PhD)

Correspondence to: DrCynthia Whitney, 1600 Clifton Road NE, Mailstop C23, Atlanta, GA 30333, USA cwhitney@cdc.gov 
Eligible children were those younger than 2 years with onset of invasive disease between Jan 1, 2001, and June 30, 2003, and children aged 2-4 years with onset between Jan 1, 2001, and May 31, 2004. According to 2002 census figures, the designated surveillance areas included about 1.7 million children younger than 5 years, including 698960 younger than 2 years and 991499 aged 2-4 years. Thus our study encompassed the equivalent of about 5 million child-years.

Cases with invasive pneumococcal disease were defined as those from whom Streptococcus pneumoniae could be isolated from usually sterile body sites. To identify such cases, we periodically contacted all clinical microbiology laboratories in the surveillance areas, and audited laboratory records at least every 6 months to ensure complete reporting. Pneumococcal isolates were sent to reference laboratories, and serotyped with the Quellung reaction. Isolates were tested at CDC, except those from Minnesota, which were tested by the local Department of Health. We classified pneumococci as vaccine-type strains if they matched the serotypes in the conjugate vaccine $(4,6 \mathrm{~B}, 9 \mathrm{~V}, 14,18 \mathrm{C}, 19 \mathrm{~F}$, and $23 \mathrm{~F})$, or as vaccinerelated strains if their serotypes were within the same serogroup as vaccine-type strains (6A, 9A, 9L, 9N, 18A, 18B, 18F, 19A, 19B, 19C, 23A, and 23B). All other pneumococcal serotypes were designated non-vaccine types.

All isolates underwent antimicrobial-susceptibility testing with the broth microdilution method at CDC, the Minnesota Department of Health, or the University of Texas Health Science Center at San Antonio. Isolates were defined as susceptible or non-susceptible according to 2006 definitions of the Clinical and Laboratory Standards Institute. ${ }^{14}$ Strains that were not susceptible to penicillin (minimum inhibitory concentration $\geq 0.12 \mu \mathrm{g} / \mathrm{mL}$ ) and to two other drug classes were regarded as multidrug-resistant strains.

Eligibility criteria were residence in an ABCS area, presence of invasive pneumococcal disease, age between 3 months and 59 months at the time of illness, and availability of isolate serotyping. Study personnel used a standard protocol to enrol children, using at least three search methods to identify telephone numbers and 15 attempts to contact the parents on different days and at various times. Children whose parents gave oral informed consent were enrolled; written consent was also obtained in San Francisco, Minnesota, and Connecticut. We excluded episodes of recurrent invasive disease to ensure that children could be enrolled in the study only once. We restricted the study to families with telephones.

For every enrolled child, a list of 15 potential controls was generated from birth-certificate registries. The list included children residing in ABCS areas who were born within 2 weeks of the enrolled child and whose reported postal zip code at birth matched the enrolled child's zip code. From this list, the child closest in age to the case was approached first. Study personnel used the same standard protocols to enrol controls as those used for children with disease. Controls were enrolled if a parent or guardian provided oral or written informed consent. We sought to enrol three controls per case.

\section{Data collection}

For both cases and controls, study personnel interviewed parents by phone to elicit household characteristics, such as number of siblings, presence of chronic medical disorders, use of breastfeeding, day-care attendance, and exposure to cigarette smoke. The aim was to gather data for the month before the date when the case child's pneumococcal culture was obtained. Interviewers were aware of case or control status. Parents were also asked to provide the name and address of their child's main health-care provider and of any other places where the child might have received vaccines. For cases and controls, we noted details of vaccination with sevenvalent pneumococcal conjugate vaccine (Prevnar, Wyeth Lederle Vaccines, Philadelphia, USA), including number of doses and dates of vaccination. Study personnel contacted these providers to obtain a medical and vaccination history for every child. In Tennessee, Georgia, and Oregon, vaccination registries were also used to verify vaccination histories. The study protocol was approved by institutional review boards at CDC and at all ABCS sites.

\section{Statistical analysis}

Data were collated and aggregated at CDC. Exclusion criteria for cases and controls included absence of verifiable written records of vaccination history, previous enrolment (or enrolment of a twin), and enrolment more than 120 days after the date when the child's pneumococcal culture was obtained. We accepted vaccination histories from providers or parents for children whose records showed no doses of pneumococcal vaccine. Analyses were done with SAS statistical software (version 9.1). We used data from the ABCS report form and $\chi^{2}$ analysis to compare characteristics of children who were enrolled with those who were not. For both cases and controls, a dose of vaccine was counted if it had been received at least 14 days before onset of illness. We used conditional logistic regression to calculate the matched odds ratio of vaccination (versus no vaccination) in cases and controls, controlling for the presence of underlying disorders and checking for colinearity and two-way interactions. To check for possible confounding, the models were repeated, with control for race, sex, day-care attendance, breastfeeding, low birthweight, and vaccination against diphtheria, tetanus, and pertussis. Vaccine effectiveness was calculated as one minus the adjusted matched odds ratio $\times 100 \%$.

We also compared vaccine schedules directly, using conditional logistic regression, controlling for underlying disorders. For these analyses we reported the odds ratios 
for disease for one schedule relative to another. For all analyses, $\mathrm{p}$ values less than 0.05 were regarded as significant.

\section{Role of the funding source}

Funding for the study was provided by CDC's Antimicrobial Resistance Working Group, CDC's Emerging Infections Program, and the US National Vaccine Program Office. These study sponsors had no role in the design or implementation of the study, analysis of data, or reporting of the results. The corresponding author had full access to all the data in the study and had final responsibility for the decision to submit for publication.

\section{Results}

We identified 1267 children with invasive pneumococcal disease during the period under study. Of these children, $485(38 \%)$ were not enrolled: $146(12 \%)$ had no isolate available for serotyping; $113(9 \%)$ could not be located; the parents of $161(13 \%)$ refused to allow them to participate; matched controls could not be enrolled for $11(1 \%)$; and $16(1 \%)$ lacked a confirmed vaccination history. A further 38 (3\%) were not enrolled for other reasons, such as identification more than 120 days after culture date or difficulty in obtaining consent because of language barriers. The remaining 782 children $(62 \%$ of the total number with invasive disease) were included in our analysis. The median age of enrolled children was about 21 months and about $40 \%$ were female (table 1). According to data from the ABCS case report form, enrolled children were of similar age to those with invasive disease who were not enrolled, but were more likely to be white $(424 / 782,54 \%$ vs $199 / 485,41 \%$, p $<0 \cdot 001)$ and were less likely to have died from their illness $(6 / 782$, $0 \cdot 8 \%$ vs $13 / 485,2 \cdot 7 \%, \mathrm{p}=0 \cdot 006)$.

The distribution of serotypes, clinical syndromes, and need for hospital treatment for invasive pneumococcal disease were much the same in enrolled and non-enrolled groups. For enrolled children with invasive disease, the most common clinical syndrome was bacteraemia without a focus (401,51\%), followed by pneumonia with bacteraemia $(237,30 \%)$, and meningitis $(65,8 \%)$. More than half $(464,59 \%)$ were treated for their pneumococcal infection as outpatients. Nearly half $(353,45 \%)$ had disease caused by one of the seven vaccine serotypes, of which serotypes 14 and $19 \mathrm{~F}$ were the most common (figure). 65 children infected with vaccine serotypes had received one or more doses of conjugate vaccine and 27 three or four doses. In those receiving three or four doses, the most common vaccine serotypes were $19 \mathrm{~F}$ (in 16) and 4 (in six). Of the total isolates, $30 \%$ were not susceptible to penicillin and $21 \%$ had decreased susceptibility to three or more antimicrobials.

We identified 8018 children as potential controls, of whom 3824 (48\%) could not be located, 1413 (18\%) refused to participate, and $182(2 \%)$ were not enrolled for

\begin{tabular}{|c|c|c|c|}
\hline & Cases $(n=782)$ & Controls ( $n=2512)$ & $\mathrm{p}$ (matched) \\
\hline Age (months) & $21.0(2-60)$ & $21.0(2-60)$ & \\
\hline \multicolumn{4}{|l|}{ Race } \\
\hline White & $455(58 \%)$ & $1740(69 \%)$ & Referent \\
\hline Black & $198(25 \%)$ & $415(17 \%)$ & $<0.0001$ \\
\hline Other/unknown & $129(17 \%)$ & $357(14 \%)$ & $<0.0001$ \\
\hline \multicolumn{4}{|l|}{ Ethnicity* } \\
\hline Hispanic & $127(16 \%)$ & $343(14 \%)$ & $0 \cdot 1060$ \\
\hline Male & $462(59 \%)$ & $1259(50 \%)$ & $<0.0001$ \\
\hline Chronic illness $\dagger$ & $88(11 \%)$ & $105(4 \%)$ & $<0.0001$ \\
\hline Immunocompromising disorder $\ddagger$ & $85(11 \%)$ & $63(3 \%)$ & $<0.0001$ \\
\hline Birthweight $<2500 \mathrm{~g}$ & $75(10 \%)$ & $169(7 \%)$ & 0.0142 \\
\hline Exposure to smoking in household & $254(33 \%)$ & $616(25 \%)$ & $<0.0001$ \\
\hline Day-care attendance & $396(51 \%)$ & $955(38 \%)$ & $<0.0001$ \\
\hline History of breastfeeding & $528(68 \%)$ & $1850(74 \%)$ & 0.0013 \\
\hline$\geq 3$ doses Haemophilus influenzae type b vaccine & $569(73 \%)$ & $1914(76 \%)$ & 0.0014 \\
\hline$\geq 3$ doses DTaP vaccine & $640(82 \%)$ & $2163(86 \%)$ & $<0.0001$ \\
\hline$\geq 1$ dose pneumococcal conjugate vaccine & $393(50 \%)$ & $1690(67 \%)$ & $<0.0001$ \\
\hline$\geq 3$ doses pneumococcal conjugate vaccine & $211(27 \%)$ & $913(36 \%)$ & $<0.0001$ \\
\hline \multicolumn{4}{|c|}{ 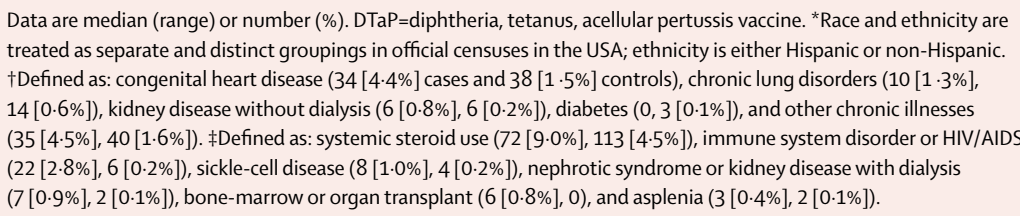 } \\
\hline
\end{tabular}

other reasons, such as no longer living in an ABCS area, having a twin already enrolled, being excluded by language, or identification more than 120 days after the date when the case's pneumococcal culture was obtained. Vaccination history could not be confirmed for $87(1 \%)$ controls. The remaining 2512 controls (29\% of those initially identified) were included in the analysis. The

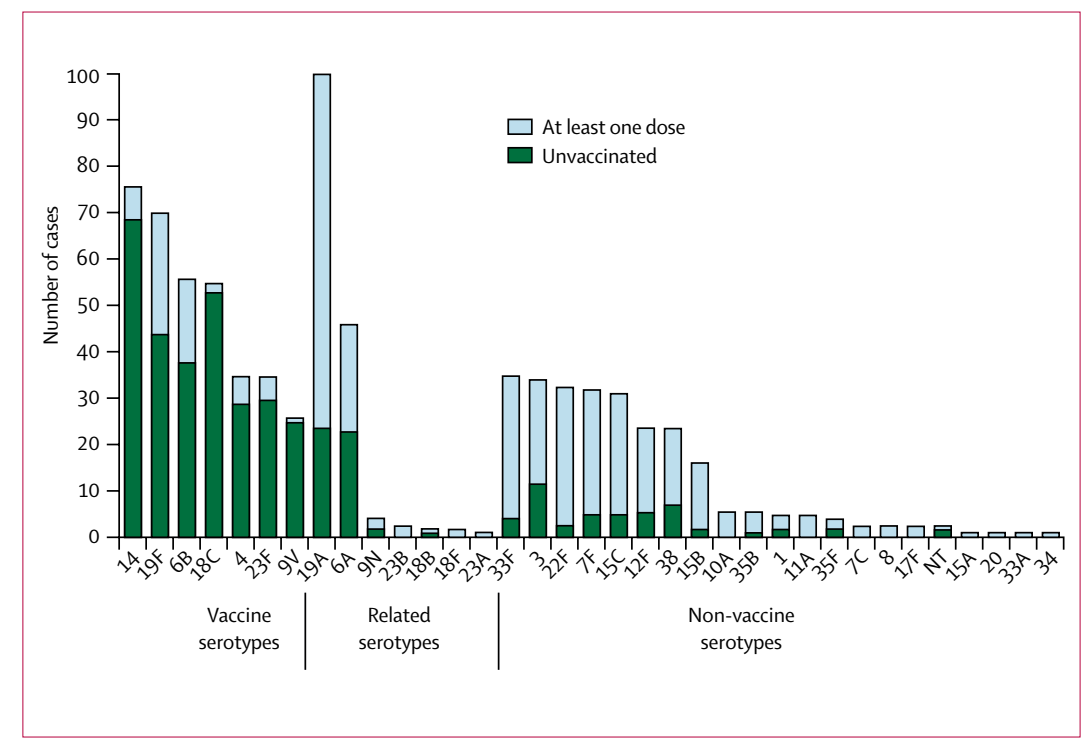

Figure: Number of cases included in the analysis by serotype and vaccination status ( $\mathrm{n}=782$ ) 


\begin{tabular}{|c|c|c|}
\hline & $\begin{array}{l}\text { Vaccine } \\
\text { effectiveness }\end{array}$ & $95 \% \mathrm{Cl}$ \\
\hline \multicolumn{3}{|l|}{ All serotypes } \\
\hline All children & $72 \%$ & $65 \%$ to $78 \%$ \\
\hline Healthy children & $71 \%$ & $63 \%$ to $78 \%$ \\
\hline Comorbid disorders & $77 \%$ & $62 \%$ to $87 \%$ \\
\hline \multicolumn{3}{|l|}{ Vaccine types } \\
\hline All children &..$*$ & \\
\hline Healthy children & $96 \%$ & $93 \%$ to $98 \%$ \\
\hline Comorbid disorders & $81 \%$ & $57 \%$ to $92 \%$ \\
\hline \multicolumn{3}{|c|}{ Vaccine-related types } \\
\hline All children & $43 \%$ & $6 \%$ to $66 \%$ \\
\hline Healthy children & $44 \%$ & $5 \%$ to $67 \%$ \\
\hline Comorbid disorders & $35 \%$ & $-151 \%$ to $83 \%$ \\
\hline \multicolumn{3}{|l|}{ Non-vaccine types } \\
\hline All children &..$\dagger$ & \\
\hline Healthy children & $-36 \%$ & $-122 \%$ to $17 \%$ \\
\hline Comorbid disorders & $77 \%$ & $32 \%$ to $92 \%$ \\
\hline \multicolumn{3}{|c|}{$\begin{array}{l}\text { *Not calculated. } \mathrm{p}=0.0014 \text { for interaction between vaccination and underlying } \\
\text { conditions in the model, so overall effectiveness is not presented. }+ \text { Not calculated } \\
p=0.0017 \text { for interaction between vaccination and underlying conditions in the } \\
\text { model. }\end{array}$} \\
\hline \multicolumn{3}{|c|}{$\begin{array}{l}\text { Table 2: Effectiveness of one or more doses of pneumococcal conjugate } \\
\text { vaccine against invasive disease in children } 3-59 \text { months of age by } \\
\text { pneumococcal serotype and the presence of chronic illnesses or } \\
\text { immunocompromising disorders }\end{array}$} \\
\hline
\end{tabular}

median number of controls was three per case (range one to ten). Interview data showed that the controls were of similar age to cases, but differed in most other characteristics (table 1).

In healthy children, the effectiveness of one or more doses of vaccine against disease caused by one of the seven vaccine serotypes was $96 \%$ (95\% CI 93-98) when we controlled for underlying disorders. This value did not change when we also controlled for race; sex; daycare attendance; low birthweight; breastfeeding; and vaccination against diphtheria, tetanus, and pertussis. Estimates of effectiveness in healthy children did not differ for those requiring hospital treatment $(96 \%, 88-99)$ versus outpatient treatment $(96 \%, 92-98)$, or for those enrolled early compared with others enrolled later in the study (97\% for 2001 cases [92-99] vs $95 \%$ for $2002-04$ cases [89-99]). Vaccine effectiveness was also similar for different syndromes; effectiveness point estimates for bacteraemia and bacteraemic pneumonia were $95 \%$ (89-98) and 98 (89-99), respectively in healthy children. Effectiveness against meningitis was $96 \%$ (95\% CI 83-99) and could not be calculated separately for healthy children and those with comorbid disorders. The vaccine was also effective against vaccine-type disease for children with underlying disorders, although less so than for healthy children (table 2).

The effect of seven-valent vaccine on the risk of disease caused by non-vaccine serotypes was modified by the presence of comorbid disorders (table 2). Vaccination did not significantly alter the risk of disease caused by nonvaccine types in healthy children, but vaccine effectiveness was just under $80 \%$ against non-vaccine-type disease in children with comorbidities. This result did not change after control for race; sex; day-care attendance; low birthweight; vaccination against diphtheria, tetanus, and pertussis; receipt of 23-valent pneumococcal vaccine (two children); household income; or antibiotic use. Effectiveness against vaccine-related serotypes was about $40 \%$ overall, and did not differ significantly in children with underlying disorders.

We also assessed the effectiveness of vaccine against invasive disease for individual serotypes (table 3). Effectiveness was lowest for serotype 19F, although the CIs overlapped for all vaccine serotypes. For vaccinerelated types, effectiveness against serotype 6A was fairly close to that of vaccine types (about $75 \%$ ), whereas the vaccine did not provide much protection against disease caused by serotype 19A. We could not assess effectiveness of vaccine against many non-vaccine types because of small numbers of cases, but vaccination was associated with a significantly higher risk of disease caused by serotype 22F.

Overall effectiveness against disease, irrespective of serotype, was around $70 \%$ (table 2), and measured effectiveness against all disease was higher in 2001 $(80 \%, 72-86)$, when vaccine serotypes accounted for $61 \%$ of cases, than in $2002-04(61 \%, 45-72)$, when only $32 \%$ of cases of disease were caused by vaccine serotypes.

\begin{tabular}{|c|c|c|c|}
\hline & $\begin{array}{l}\text { Number of } \\
\text { discordant sets* }\end{array}$ & $\begin{array}{l}\text { Vaccine } \\
\text { effectiveness }\end{array}$ & $95 \% \mathrm{Cl}$ \\
\hline \multicolumn{4}{|c|}{ Vaccine types } \\
\hline 4 & 19 & $93 \%$ & $65 \%$ to $99 \%$ \\
\hline $6 B$ & 32 & $94 \%$ & $77 \%$ to $98 \%$ \\
\hline $9 \mathrm{~V}$ & 20 & $100 \%$ & $88 \%$ to $100 \%$ \\
\hline 14 & 47 & $94 \%$ & $81 \%$ to $98 \%$ \\
\hline $18 C$ & 30 & $97 \%$ & $85 \%$ to $99 \%$ \\
\hline $19 F$ & 34 & $87 \%$ & $65 \%$ to $95 \%$ \\
\hline $23 \mathrm{~F}$ & 18 & $98 \%$ & $80 \%$ to $100 \%$ \\
\hline \multicolumn{4}{|c|}{ Vaccine-related types } \\
\hline $6 \mathrm{~A}$ & 26 & $76 \%$ & $39 \%$ to $90 \%$ \\
\hline $19 A$ & 46 & $26 \%$ & $-45 \%$ to $62 \%$ \\
\hline \multicolumn{4}{|c|}{ Non-vaccine types } \\
\hline $33 \mathrm{~F}$ & 11 & $22 \%$ & $-206 \%$ to $80 \%$ \\
\hline 3 & 14 & $30 \%$ & $-131 \%$ to $79 \%$ \\
\hline $22 \mathrm{~F}$ & 13 & $-899 \%$ & $-8302 \%$ to $-19 \%$ \\
\hline $7 F$ & 12 & $-22 \%$ & $-444 \%$ to $73 \%$ \\
\hline $15 C$ & 10 & -55 & $-607 \%$ to $66 \%$ \\
\hline \multicolumn{4}{|c|}{$\begin{array}{l}\text { *Only case-control sets discordant for exposure of interest (vaccination) } \\
\text { contribute to calculations in matched analysis. }\end{array}$} \\
\hline \multicolumn{4}{|c|}{$\begin{array}{l}\text { Table 3: Effectiveness of one or more doses of pneumococcal conjugate } \\
\text { vaccine against invasive disease in children aged 3-59 months by } \\
\text { pneumococcal serotype }\end{array}$} \\
\hline
\end{tabular}


Vaccine effectiveness was $76 \%$ (63-85) against disease caused by strains not susceptible to penicillin and 77\% (62-86) against multidrug-resistant strains.

Compared with no vaccine, the effectiveness of one dose given at 7 months of age or earlier against vaccine serotypes was just over 70\% (table 4). When stratified by time since vaccination, one dose was protective for up to 6 months $(84 \%, 58-94)$ but we could not show protection 6 months or more after vaccination $(33 \%,-143$ to 81 ). Compared with no vaccine, point estimates for effectiveness of two, three, or four doses when given on an infant schedule were close to each other, with widely overlapping $\mathrm{CI}$ and were more effective than a single dose (table 4). Effectiveness of two, three, and four-dose schedules was similar up to 6 months after vaccination (97\% [87-99] for 2 doses, 100\% [96-100] for 3 doses, and $100 \%$ [58-100] for 4 doses) and 6 or more months following vaccination (95\% [71-99] for 2 doses, 87\% [64-95] for 3 doses, and 100\% [93-100] for 4 doses). We examined vaccine effectiveness in children starting their series of doses late in their first year or after their first birthday, and showed that catch-up schedules were also highly effective (table 4).

In a comparison of a schedule of three doses given at up to 7 months of age, with a four-dose infant schedule (three doses up to 7 months and a fourth dose at 12-16 months), the four-dose schedule significantly reduced risk of disease caused by vaccine serotypes (matched odds ratio $0,0-0 \cdot 87$; table 5 ). We did not identify significant differences on direct comparisons between other schedules of two, three, and four doses, although small numbers of children vaccinated on some of these schedules restricted our ability to make comparisons.

\section{Discussion}

The seven-valent conjugate vaccine was protective in children 3-59 months old against disease caused by all seven serotypes contained in the vaccine, against disease caused by antibiotic-resistant strains, and against all invasive pneumococcal disease irrespective of serotype. Our results accord with findings of earlier clinical trials in which seven-valent or nine-valent conjugate vaccine formulations were given in highly controlled settings in which infants were vaccinated on three-dose or four-dose schedules..$^{1-4}$ In these clinical trials, effectiveness against disease caused by vaccine serotypes ranged from $65 \%$ in children with HIV infection in South Africa ${ }^{3}$ to $94 \%$ in child members of a health-care system in northern California. ${ }^{1}$ In South Africa, lower efficacy was seen in children infected with HIV than in those not infected; ${ }^{3}$ we also recorded lower effectiveness in those with chronic illnesses than in healthy children. A trial of the ninevalent vaccine in The Gambia ${ }^{4}$ proved its efficacy for serotypes 5, 14, and 23F; we showed that the seven-valent vaccine is effective against disease caused by all seven individual serotypes represented in the vaccine, as well

\begin{tabular}{|c|c|c|}
\hline & Effectiveness & $95 \% \mathrm{Cl}$ \\
\hline \multicolumn{3}{|l|}{ Infant schedules* } \\
\hline 1 dose $\leq 7$ months & $73 \%$ & $43 \%$ to $87 \%$ \\
\hline 2 doses $\leq 7$ months & $96 \%$ & $88 \%$ to $99 \%$ \\
\hline 3 doses $\leq 7$ months & $95 \%$ & $88 \%$ to $98 \%$ \\
\hline 1 dose $\leq 7$ months, 1 dose $8-11$ months, 1 dose $12-16$ months $\dagger$ & $100 \%$ & $88 \%$ to $100 \%$ \\
\hline 2 doses $\leq 7$ months, 1 dose $12-16$ monthst & $98 \%$ & $75 \%$ to $100 \%$ \\
\hline 3 doses $\leq 7$ months, 1 dose $12-16$ months $\dagger$ & $100 \%$ & $94 \%$ to $100 \%$ \\
\hline 1 dose $7-11$ months, 2 doses $12-16$ months $\dagger$ & $98 \%$ & $83 \%$ to $100 \%$ \\
\hline \multicolumn{3}{|l|}{ Toddler schedules* } \\
\hline 1 dose $12-23$ months & $93 \%$ & $68 \%$ to $98 \%$ \\
\hline 2 doses $12-23$ months $\dagger$ & $96 \%$ & $81 \%$ to $99 \%$ \\
\hline 1 dose $\geq 24$ months $\dagger$ & $94 \%$ & $49 \%$ to $99 \%$ \\
\hline \multicolumn{3}{|c|}{$\begin{array}{l}\text { *Vaccine schedules, by months of age at time of doses, are mutually exclusive. †Based on vaccination schedules } \\
\text { recommended by the Advisory Committee on Immunization Practices. }{ }^{7} \text { We could not assess two recommended } \\
\text { schedules (two doses } 7-11 \text { months plus one dose } 12-16 \text { months, and two doses at } 24 \text { months or later) because } \\
\text { insufficient numbers of cases and controls were vaccinated on those schedules. }\end{array}$} \\
\hline
\end{tabular}

as serotype 6A, which is structurally similar to vaccine serotype 6B. Our findings also show that the conjugate vaccine is highly effective for prevention of invasive disease in children who have been vaccinated on different catch-up schedules and in children who received fewer than the recommended number of doses.

Our data suggest that several schedules afford good individual protection, although we could not show protection for one dose given before 6 months of age against episodes of illness occurring after 6 months or more. The four-dose schedule recommended in the USA has disadvantages because the conjugate vaccine is expensive, and health-care providers and parents prefer that infants receive as few injections as possible. ${ }^{15}$ Furthermore, schedules for routine infant immunisations in many parts of the world could more easily accommodate a three-dose series, with either three doses in the first 6 months of life or two doses within 6 months, followed by a booster at 1 year of age. In our study, point estimates of vaccine effectiveness for schedules with two, three, and four doses seemed very similar when assessed against no vaccination. We were unable to identify differences in protective effect of most schedules, although the risk of disease was reduced more by a schedule of three doses within 7 months, plus a booster than by three infant doses without a booster. A schedule of two doses within 7 months, plus a booster, as has been adopted for routine use in the UK and elsewhere, was highly protective compared with no vaccine, but too few children enrolled in our study were vaccinated on this schedule for us to compare its effectiveness directly with other schedules. Clinical trials of the nine-valent formulation in Africa showed that a series of three doses given early in infancy was effective against invasive disease and pneumonia., 


\begin{tabular}{|c|c|c|c|}
\hline & \multicolumn{3}{|c|}{ Matched, adjusted odds ratio $(95 \% \mathrm{Cl})$} \\
\hline & 3 doses $\leq 7$ months, no booster* & 2 doses $\leq 7$ months, 1 dose $12-16$ months ${ }^{*} \dagger$ & 2 doses $\leq 7$ months, no booster* \\
\hline 3 doses $\leq 7$ months, 1 dose $12-16$ months & $0(0,0.87) \ddagger$ & $0(0-10 \cdot 1)$ & $0(0-1 \cdot 54) \varsigma$ \\
\hline 3 doses $\leq 7$ months, no booster & & $1.5(0.15-14 \cdot 6)$ & $1.5(0.54-4.35)$ \\
\hline 2 doses $\leq 7$ months, 1 dose $12-16$ months $\dagger$ & & & $0.85(0.08-9 \cdot 1)$ \\
\hline
\end{tabular}

An important component of the success of pneumococcal conjugate vaccine has been its ability to prevent the acquisition of carriage of vaccine serotypes in the nasopharynx of vaccinated children. ${ }^{10,16,17}$ The vaccine thereby reduces transmission of vaccine-type pneumococci, and protects unvaccinated children and adults from disease, thus producing indirect benefits from vaccination known as herd immunity. ${ }^{10,16,17}$ Protection against pneumonia, otitis media, and carriage of vaccine-type strains might require higher antibody titres, and therefore more doses, than does prevention of invasive pneumococcal disease. ${ }^{18-20}$ Studies have shown a high rate of production of antibodies after vaccination with two doses before 6 months of age, with a booster dose at 11 or 12 months..$^{21,22}$ The antibody response after two doses at or before 6 months of age with a booster was much the same as it was after three doses with a booster. ${ }^{23}$

A rise in vaccine use during our study caused an increase in herd immunity and a reduction in the number of children with vaccine-type disease available for enrolment as the study progressed. In the USA, the estimated number of cases of invasive disease in children younger than 5 years fell from an average of 17240 per year in $1998-99$ to 4454 in $2003 .{ }^{24}$ We did not show any difference in effectiveness against vaccine serotypes with time, however, which suggests that herd effects did not confound our ability to measure specific vaccine effectiveness. Effectiveness of the vaccine against all invasive disease, without regard to serotype, diminished in that non-vaccine types accounted for a larger proportion of cases later in the study. As the amount of vaccine-type disease has fallen, a small increase has occurred in the rate of disease caused by serotypes not contained in the conjugate vaccine-in particular disease caused by 19A..$^{11,25}$ Serotype 19A was the most common cause of disease in children enrolled in our study. We previously showed that many isolates of this serotype are resistant to several antibiotics. ${ }^{25}$ Our results indicated that conjugate vaccine did not protect against disease caused by serotype 19A, even though this serotype is structurally similar to vaccine serotype 19F. In a Finnish trial, ${ }^{6}$ vaccination did not prevent otitis media caused by serotype 19A. Since the conjugate vaccine does not provide cross-protection against this important serotype, an antigen targeting serotype 19A should be included in future vaccine formulations.
Overall, our findings indicated that vaccinated children were not at greater risk of disease caused by non-vaccine serotypes than were unvaccinated children in our study. However, vaccinated children did have a higher risk of disease caused by the pneumococcal serotype 22F.

We showed that the seven-valent conjugate vaccine was effective against disease caused by antibioticresistant strains; this finding lends support to clinical trial results from South Africa ${ }^{3}$ and surveillance data from the USA, suggesting that vaccination reduces infections caused by resistant pneumococci. ${ }^{26-28}$ The vaccine's effect on resistant infections is not surprising, since five of the seven vaccine serotypes account for most disease caused by resistant pneumococci, and that serotype 6A, a vaccine-related serotype for which the vaccine provides protection, is also commonly resistant. ${ }^{29}$ Before the vaccine was licensed, $78 \%$ of invasive infections in the USA that were attributable to penicillin-resistant organisms were caused by serotypes contained in the seven-valent conjugate vaccine, ${ }^{30}$ and 18 of 26 multidrug-resistant pneumococcal clones reported to cause disease worldwide were vaccine serotypes. $^{31}$

A limitation of case-control studies is their observational nature, which can lead to bias and confounding. We took care to avoid bias in selection of controls, by using rigorous methods to locate and enrol control children. Our participation rates were acceptable. We also controlled for many possible confounders such as known risk factors for disease, and our estimates of effectiveness were not altered by access to vaccines (eg, receipt of diphtheria, tetanus, and pertussis vaccine). Nonetheless, our finding of $77 \%$ effectiveness against disease caused by non-vaccine types in children with comorbid disorders was surprising, and might indicate a chance finding or the effect of an unidentified confounding factor. The large size of our study was a strength, and allowed us to assess the effectiveness of individual vaccine serotypes and several different dosing schedules. Nonetheless, our ability to directly compare the protection provided by some schedules relative to others was limited by the small numbers of children who had been vaccinated on some schedules.

Our results indicate that the seven-valent pneumococcal conjugate vaccine has been very effective in practice in the USA. This information adds to evidence indicating that pneumococcal conjugate vaccines have 
the potential to greatly reduce the 800000 to 1 million deaths of children from pneumococcal disease every year. $^{32}$ The next challenge is to ensure that conjugate vaccines become part of routine immunisation in more places, especially in developing countries where most pneumococcal deaths in young children occur.

\section{Contributors}

All the authors participated in the design, implementation, analysis, and interpretation of the study. C G Whitney was the overall principal investigator, drafted the report, and secured funding. T Pilishvili and E R Zell oversaw data management and conducted the analysis. M M Farley, W Schaffner, A S Craig, R Lynfield, A-C Nyquist, K A Gershman, M Vazquez, N M Bennett, A Reingold, M P Glode, and A Thomas were principal investigators for individual ABCS sites and secured funding. J H Jorgensen and B Beall directed the microbiological laboratory work. A Schuchat was chief of the Respiratory Diseases Branch, overseeing ABCS activities and laboratory work and securing funding for $\mathrm{ABCS}$.

\section{Conflict of interest statement}

We declare that we have no conflict of interest, apart from the following: Wyeth paid travel costs for M Farley to two meetings to present data on the epidemiology of pneumococcal disease. A-C Nyquist was a member of the Wyeth Lederle speakers bureau. E Zell owns a small amount of stock in Merck, Pfizer, and Johnson \& Johnson. N Bennett serves on a Merck advisory board for zoster vaccine and was funded by Wyeth to give a presentation on pneumococcal disease.

\section{Acknowledgments}

We thank the following people for their contributions to data collection, analysis, laboratory work, or study direction: James Howgate, Laura Rainer, Kathryn E Arnold, and Wendy Baughman (Georgia Emerging Infections Program); Catherine Muehlenbein, Maria Thomas, and Eugene D Shapiro (Yale University); James Hadler, Nancy L Barrett (Connecticut Emerging Infections Program, ABCS Project); Pam Daily, Susan Brooks, and Emma Lucas (California Emerging Infections Program); Elizabeth Esterl, Molly Byrne, and Hazel Senz (The Children's Hospital, Denver, CO); Mathew Finke (Colorado Department of Public Health and Environment); Pamala Gahr, Catherine Lexau, Billi Juni, Anita Glennen, Bonnie Koziol, Gertrud Kupferschmidt, Kerry MacInnes, and Darla Tuil (Minnesota Department of Health); Bridget Anderson, Shelley Zansky, Christine Long, and Dina Hoefer (New York Emerging Infections Program); Karen Stefonek, Linda Duke, and Michelle Barber (Oregon Department of Human Services); Brenda Barnes, Belinda Redd, Jane Conners, Patty Sackett, Jan Roulstone, Terri McMinn, and Selma Archer (Tennessee Emerging Infections Program); Chris Van Beneden, Tami Skoff, Carolyn Wright, Sandra McCoy, Richard Facklam, and Delois Jackson (Centers for Disease Control and Prevention); and M Leticia McElmeel, Letitia Fulcher, Christa Trippy, and Sharon Crawford (University of Texas Health Sciences Center at San Antonio).

\section{References}

1 Black S, Shinefield H, Fireman B, et al. Efficacy, safety and immunogenicity of heptavalent pneumococcal conjugate vaccine in children. Pediatr Infect Dis J 2000; 19: 187-95.

2 O’Brien KL, Moulton LH, Reid R, et al. Efficacy and safety of sevenvalent conjugate pneumococcal vaccine in American Indian children: group randomised trial. Lancet 2003; 362: 355-61.

3 Klugman KP, Madhi SA, Huebner RE, et al. A trial of a 9-valent pneumococcal conjugate vaccine in children with and those without HIV infection. N Engl J Med 2003; 349: 1341-48.

4 Cutts F, Zaman S, Enwere G, et al. Efficacy of nine-valent pneumococcal conjugate vaccine against pneumonia and invasive pneumococcal disease in The Gambia: randomised, double-blind, placebo-controlled trial. Lancet 2005; 365: 1139-46.

5 Black SB, Shinefield HR, Ling S, et al. Effectiveness of heptavalen pneumococcal conjugate vaccine in children younger than five years of age for prevention of pneumonia. Pediatr Infect Dis J 2002; 21: $810-15$
6 Eskola J, Kilpi T, Palmu A, et al. Efficacy of a pneumococcal conjugate vaccine against acute otitis media. N Engl J Med 2001; 344: 403-09.

7 Centers for Disease Control and Prevention. Prevention of pneumococcal disease among infants and young children: recommendations of the Advisory Committee on Immunization Practices. MMWR Morb Mortal Wkly Rep 2000; 49: 1-35.

8 American Academy of Pediatrics, Committee on Infectious Diseases. Recommendations for the prevention of pneumococcal infections, including the use of pneumococcal conjugate vaccine (Prevnar), pneumococcal polysaccharide vaccine, and antibiotic prophylaxis (RE9960). Pediatrics 2000; 106: 362-66.

9 Centers for Disease Control and Prevention. Notice to readers: updated recommendations on the use of pneumococcal conjugate vaccine in a setting of vaccine shortage-Advisory Committee on Immunization Practices. MMWR Morb Mortal Wkly Rep 2001; 50: $1140-42$.

10 Flannery B, Schrag S, Bennett NM, et al. Impact of childhood vaccination on racial disparities in invasive Streptococcus pneumoniae infections. JAMA 2004; 291: 2197-203.

11 Kaplan SL, Mason EOJ, Wald ER, et al. Decrease of invasive pneumococcal infections in children among 8 children's hospitals in the United States after the introduction of the 7 -valent pneumococcal conjugate vaccine. Pediatrics 2004; 113: 443-49.

12 Black S, Shinefield H, Baxter R, et al. Postlicensure surveillance for pneumococcal invasive disease after use of heptavalent pneumococcal conjugate vaccine in Northern California Kaiser Permanente. Pediatr Infect Dis J 2004; 23: 485-89.

13 Schuchat A, Hilger T, Zell E, et al. Active Bacterial Core Surveillance of the Emerging Infections Program Network. Emerg Infect Dis 2001; 7: 92-99.

14 Clinical and Laboratory Standards Institute. Performance standards for antimicrobial susceptibility testing. Sixteenth informational supplement (M100-S16). Wayne, PA: Clinical and Laboratory Standards Institute; 2006.

15 Woodin KA, Rodewald LE, Humiston SG, Carges MS, Schaffer SJ, Szilagyi PG. Physician and parent opinions. Are children becoming pincushions from immunizations? Arch Pediatr Adolesc Med 1995; 149: 845-49.

16 Lexau CA, Lynfield R, Danila R, et al. Changing epidemiology of invasive pneumococcal disease among older adults in the era of pediatric pneumococcal conjugate vaccine. JAMA 2005; 294: 2043-51.

17 Flannery BL, Heffernan RT, Harrison LH, et al. Changes in invasive pneumococcal disease among HIV-infected adults living in the era of childhood pneumococcal immunization. Ann Intern Med 2006; 144: 1-9.

18 Jodar L, Butler J, Carlone G, et al. Serological criteria for evaluation and licensure of new pneumococcal conjugate vaccine formulations for use in infants. Vaccine 2003; 21: 3265-72.

19 Jokinen J, Ahman H, Kilpi TM, Makela PH, Kayhty MH. Concentration of antipneumococcal antibodies as a serological correlate of protection: an application to acute otitis media. $J$ Infect Dis 2004; 190: $545-50$.

20 Dagan R, Givon-Lavi N, Fraser D, Lipsitch M, Siber GR, Kohberger R. Serum serotype-specific pneumococcal anticapsular immunoglobulin $\mathrm{G}$ concentrations after immunization with a 9-valent conjugate pneumococcal vaccine correlate with nasopharyngeal acquisition of pneumococcus. J Infect Dis 2005; 192: 367-76.

21 Esposito S, Pugni L, Bosis S, et al. Immunogenicity, safety and tolerability of heptavalent pneumococcal conjugate vaccine administered at 3, 5 and 11 months post-natally to pre- and fullterm infants. Vaccine 2005; 23: 1703-08.

22 Kayhty H, Ahman H, Eriksson K, Sorberg M, Nilsson L. Immunogenicity and tolerability of a heptavalent pneumococcal conjugate vaccine administered at 3, 5 and 12 months of age. Pediatr Infect Dis J 2005; 24: 108-14.

23 Goldblatt D, Southern J, Ashton L, et al. Immunogenicity and boosting after a reduced number of doses of a pneumococcal conjugate vaccine in infants and toddlers. Pediatr Infect Dis J 2006; 25: 312-19. 
24 Centers for Disease Control and Prevention. Direct and Indirect effects of routine vaccination of children with 7-valent pneumococcal conjugate vaccine on incidence of invasive pneumococcal disease-United States, 1998-2003. MMWR Morb Mortal Wkly Rep 2005; 54: 893-97.

25 Pai R, Moore MR, Pilishvili T, et al. Postvaccine genetic structure of Streptococcus pneumoniae serotype 19A from children in the United States. J Infect Dis 2005; 192: 1988-95.

26 Kyaw MH, Lynfield R, Schaffner W, et al. Effect of pneumococcal conjugate vaccine introduction on drug-resistant Streptococcus pneumoniae. N Engl J Med 2006; 354: 1455-63.

27 Talbot T, Poehling K, Hartert R, et al. Reduction in high rates of antibiotic-nonsusceptible invasive pneumococcal disease in Tennessee after introduction of the pneumococcal conjugate vaccine. Clin Infect Dis 2004; 39: 641-48.

28 Doern GV, Richter SS, Miller A, et al. Antimicrobial resistance among Streptococcus pneumoniae in the United States: have we begun to turn the corner on resistance to certain antimicrobial classes? Clin Infect Dis 2005; 41: 139-48.
29 Whitney CG, Klugman KP. Vaccines as tools against resistance: the example of pneumococcal conjugate vaccine.

Semin Pediatr Infect Dis 2004; 15: 86-93.

30 Whitney CG, Farley MM, Hadler J, et al. Increasing prevalence of multidrug-resistant Streptococcus pneumoniae in the United States. N Engl J Med 2000; 343: 1917-24.

31 Pneumococcal Molecular Epidemiology Network. Pneumococcal Molecular Epidemiology Network. http://www.sph.emory.edu/ PMEN/index.html. (accessed Aug 6, 2005).

32 World Health Organization. Pneumococcal vaccines. Wkly Epidemiol Record 2003; 14: 110-19. 